\title{
LA REVOLUCIÓN FINTECH EN LOS MEDIOS DE PAGO: SITUACIÓN ACTUAL Y PERSPECTIVAS
}

\author{
Gloria M. Soto Pacheco (Universidad de Murcia) ${ }^{1}$ \\ María Teresa Botía Martínez (Universidad de Murcia) ${ }^{2}$
}

\section{Resumen:}

La tecnología y la nueva era digital están suponiendo grandes transformaciones en todos los sectores de la economía. En el ámbito financiero, la aplicación de la tecnología da como resultado lo que se conoce como fintech. Aunque la combinación de finanzas y tecnología nos lleva acompañando desde hace décadas, en los últimos años ha adquirido una gran relevancia debido a la rapidez con que se suceden las innovaciones como consecuencia de la democratización de la digitalización, esto es, del acceso masivo y barato a las nuevas tecnologías por empresas y consumidores. El fenómeno fintech abarca una gran variedad de áreas, también denominadas verticales, que incluyen desde los medios de pago hasta la financiación alternativa, pasando por las criptomonedas o el big data, por citar algunos ejemplos.

Este trabajo se centra en las repercusiones fintech en el segmento de los medios de pago, en donde viene registrándose toda una revolución. El uso del efectivo se está reduciendo debido a la aparición de nuevas y variadas alternativas como los monederos digitales, las plataformas P2P y otras alternativas cuyo uso está menos generalizado, como los pagos mediante factura telefónica. Realizamos un detallado análisis sobre estas innovaciones explicando su funcionamiento, los principales operadores y su evolución. También se abordan las perspectivas futuras, al objeto de conocer qué nos depara el futuro y las posibles implicaciones y adaptaciones de los cambios que están por venir relacionados con la tecnología blockchain, la inteligencia artificial, el internet de las cosas o la computación cuántica.

Palabras clave: fintech, medios de pago, dinero, digitalización.

Códigos JEL: D14, E40, G20.

\section{Abstract:}

Technology and the new digital era are motivating huge changes in all sectors of the economy. In the financial area, the application of technology results in what is known as fintech. Although the combination of finance and technology has been accompanying us for decades, in recent years it has gained increased relevance due to the rapidity with which innovations appear as a result of the democratization of digitalization, that is, the massive and cheap access to new technologies by companies and consumers. The fintech phenomenon encompasses a wide variety of areas, also called verticals, including means of payment, alternative financing, financial advisory, cryptocurrencies, blockchain, insurtech and big data, among others.

This study focuses on the impact of fintech on payment methods, where a real revolution is taking place. The use of cash is declining due to the emergence of new and varied methods such as digital wallets, P2P platforms and other alternatives whose use is less widespread, such as direct carrier billing. The study provides a detailed analysis of these innovations explaining their operation, the main agents and their evolution. Future perspectives are also addressed in

\footnotetext{
1 gsoto@um.es, Universidad de Murcia.

2 mteresa.botia@um.es, Universidad de Murcia.

Recibido: 25 de noviembre de 2019. Aceptado: 25 de septiembre de 2020.
} 
order to know what the future holds and the possible implications and adaptations of the changes that are to come, related to blockchain technology, artificial intelligence, the Internet of things or quantum computing.

Key words: fintech, payment methods, money, digitalization.

JEL Codes: D14, E40, G20.

\section{INTRODUCCIÓN}

El término fintech procede de la abreviatura de financial technology, y a pesar de que a veces se alude a él como si fuese un fenómeno o un término nuevo, lo cierto es que no es así. La digitalización del sector financiero y la aplicación de las nuevas tecnologías al mismo es un proceso que viene desarrollándose desde la aparición de los primeros cajeros automáticos a finales de la década de 1960 (CNMC, 2018). Más adelante, con el surgimiento de Internet, la adopción e implementación de nuevos servicios en el sector financiero se fue intensificando a la par que nos adentrábamos en siglo XXI. Y el ritmo vertiginoso al que crece en los últimos años el fenómeno fintech encuentra su principal explicación en la "democratización tecnológica de esa digitalización”, es decir, el libre acceso a estas nuevas tecnologías, su uso masivo y la capacidad con la que cuenta en la actualidad cualquier usuario para acceder a la información (Carbó, 2017). A ello hay que unir cambios regulatorios que han propiciado la aparición de nuevos competidores en el ámbito financiero (en el caso de la Unión Europea, con la nueva Directiva de Servicios de Pago, PSD2, que entró en vigor en enero de 2018) así como el surgimiento de nuevas tecnologías que amplían el abanico de posibilidades y aplicaciones.

Desde hace años, al fenómeno fintech se le ha considerado toda una revolución. Por ejemplo, Kauffman y Ma (2015, p.261) ya se referían a él como "global fintech revolution” y, según Deloitte (2016), el desarrollo del fintech provocaría un gran impacto en la experiencia de los consumidores y cambiaría tanto la estructura competitiva como el ecosistema de los servicios financieros. Los datos apoyan estas apreciaciones. Como se muestra en la figura 1, el valor de las inversiones fintech a nivel mundial ha pasado de representar 1.100 millones de dólares en 2008 a situarse en 69.500 millones de dólares en 2019.

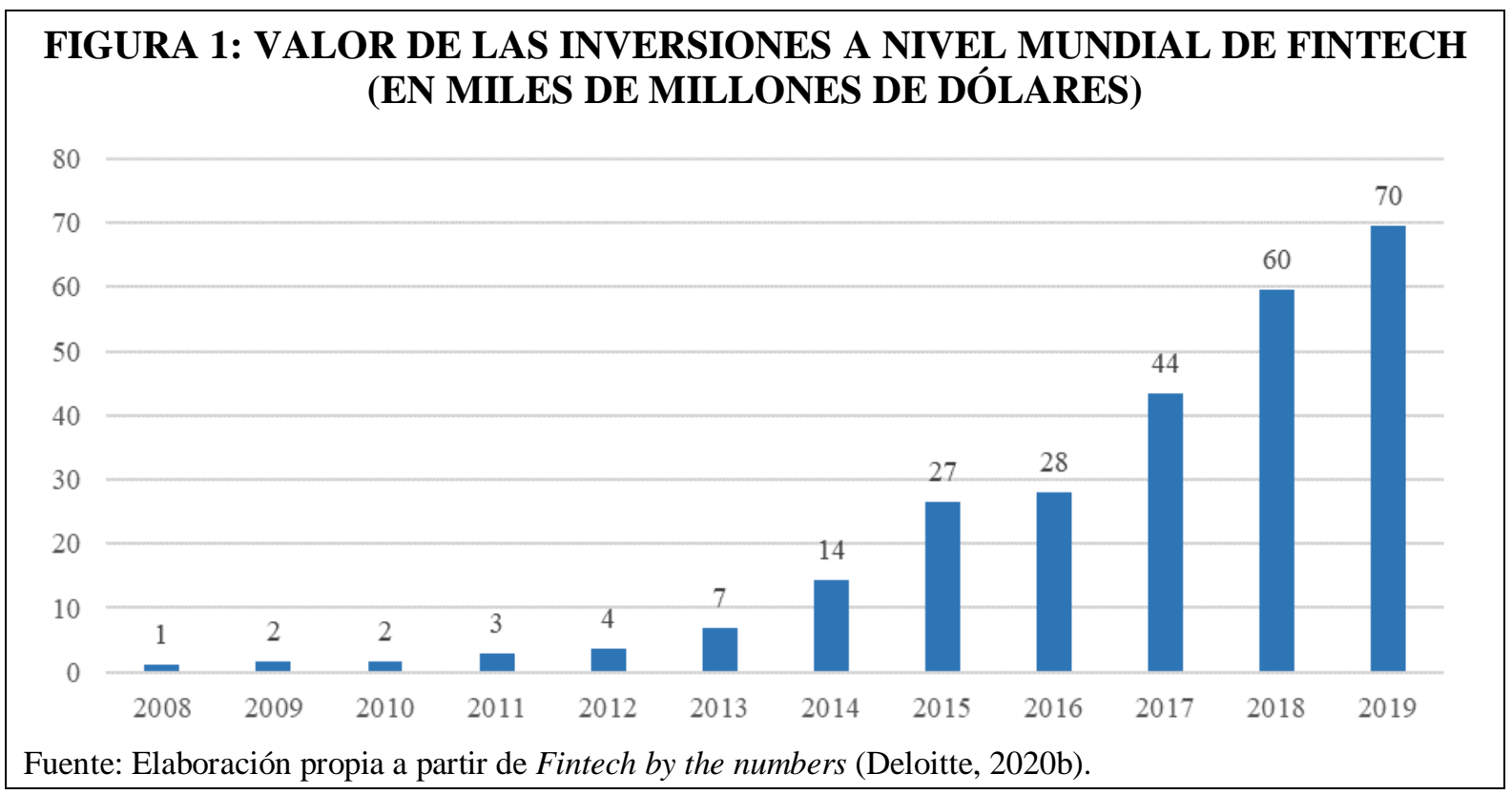


Aunque no existe una definición comúnmente aceptada de fintech, todas comparten un nexo común, y es la idea de innovación, de cambio y mejora en el sector financiero. Así, existen autores que establecen definiciones amplias, por ejemplo, según Schindler (2017, p.2) fintech es "la innovación financiera tecnológica que podría resultar en nuevos modelos de negocio, aplicaciones, procesos, productos o servicios con un efecto importante en los mercados e instituciones financieras y en la provisión de servicios financieros”, o Schueffel (2016, p. 15), que concreta el fintech como "una nueva industria financiera que aplica tecnología para mejorar las actividades financieras”. Sin embargo, también se puede definir en un sentido más concreto como "la aplicación disruptiva de los nuevos desarrollos en TIC, por parte de las empresas financieras ya instaladas y de entrantes en el sector financiero, que buscan atender directamente necesidades no satisfechas o mejorar la forma en la que se satisfacen actualmente algunas necesidades financieras” (CNMC, 2018, p.99).

Siguiendo la clasificación realizada por la Asociación Española de Fintech e Insurtech (AEFI, 2017) ${ }^{3}$, se pueden distinguir los siguientes verticales de fintech e insurtech agrupando a las empresas según las diferentes líneas de negocio que desarrollen:

- Asesoramiento y gestión patrimonial: incluye a empresas que prestan servicios relacionados con la gestión de las inversiones buscando brindar un servicio más personalizado y accesible a un mayor número de clientes. Dentro de esta categoría se incluirían empresas como redes sociales de inversión, empresas de asesoramiento y gestión automatizada y plataformas de negociación

- Finanzas personales: abarca negocios que proporcionan a sus clientes información y pronósticos sobre sus gastos financieros y su dinero disponible conectando las diferentes cuentas bancarias de las que se dispongan, es decir, empresas tales como comparadores de productos financieros y de gestión eficiente de las finanzas.

- Financiación alternativa: este vertical engloba tanto a las entidades que facilitan la concesión de créditos con rapidez y sin necesidad de garantía a los particulares a través de plataformas informáticas, que son los conocidos como Préstamos Rápidos Online o plataformas de préstamos al consumo; el crowdfunding, que son los métodos de financiación de proyectos basados en una red de financiación colectiva online; por último, se incluyen en esta categoría ciertas plataformas de financiación para empresas y los préstamos concedidos entre particulares (P2P).

- Servicios transaccionales/divisas: esta categoría comprende las empresas que ofrecen menor coste y más flexibilidad en operaciones con divisas extranjeras tratando de obtener las mejores tasas de cambio y sin costes procedentes de la intermediación bancaria.

- Medios de pago: se incluyen las entidades cuyo objeto principal se basa en proporcionar servicios de pago generalmente online a través de plataformas electrónicas y dispositivos móviles.

- Neobanks y challenger banks: en esta vertical se encuentran los bancos que hacen un uso extensivo de la tecnología ofreciendo servicios bancarios a través smartphones y plataformas digitales; es decir, se trata de bancos totalmente digitales que ofrecen una amplia gama de servicios más allá de los tradicionales (cuentas corrientes, de depósito, tarjetas de créditos, asesoramiento, préstamos, etc.) a un menor coste y de

\footnotetext{
${ }^{3}$ La clasificación no es idéntica a la que se presenta en AEFI (2017) porque ésta realiza una separación de varias especialidades de crowdfunding que luego no se ha mantenido, al tiempo que han surgido otras verticales como los Neobanks y challenger banks que se han incorporado a la lista de verticales que se ofrece en la página web de la asociación. Las explicaciones de las verticales sí respetan lo establecido en AEFI (2017).
} 
forma innovadora, alejándose del modelo de relación con el cliente a través de una sucursal bancaria (Bank for International Settlements [BIS], 2018).

- Infraestructura financiera: incluye a las empresas dedicadas a la innovación y las mejoras relacionadas con la tecnología que proporcionan productos y servicios a empresas fintech o a entidades financieras, en definitiva, empresas que aportan mejoras en la prestación de servicios financieros, pero sin estar en contacto directo con el cliente final.

- Criptomonedas y blockchain: las criptomonedas son un medio de intercambio digital descentralizado no respaldado por los gobiernos; la tecnología blockchain, o de cadena de bloques, surgió con la primera moneda virtual, el Bitcoin, y es un mecanismo que permite que los participantes mantengan y editen bases de datos de forma descentralizada, sin intermediarios, y que puede aplicarse a transacciones, contratos, activos, identidades $\mathrm{y}$, en general, a cualquier aspecto que se pueda describir en formato digital.

- Insurtech: supone la aplicación de avances tecnológicos como big data y aplicaciones móviles y la automatización en el sector de las aseguradoras, dando lugar a nuevos servicios como seguros entre particulares (P2P), comparadores web y agregadores financieros al servicio de seguros o seguros a demanda relacionados con transacciones específicas, que permiten negociar condiciones más favorables y personalizadas.

- Identificación online de clientes: representa a las empresas dedicadas a desarrollar nuevas tecnologías que faciliten la identificación de clientes en las diferentes transacciones que estos realizan, en otras palabras, tecnologías que permiten verificar que una persona es quien dice ser proporcionando así una mayor transparencia y seguridad a la hora de realizar negocios.

- Big data: esta vertical abarca todas aquellas empresas que recogen, almacenan, gestionan y analizan grandes volúmenes de datos, generando un valor añadido y prestando servicios relacionados con el uso de estos datos.

En la figura 2 se puede apreciar como dentro del sector fintech el segmento de mayor relevancia son los medios de pago. Se espera que el valor total de las transacciones mundiales de medios de pagos digitales muestre una tasa de crecimiento anual en el período de 2017 a 2024 en torno al 18,5 por cien, lo que resultaría en un valor cercano a los 8,3 billones de dólares para el año 2024 (Statista, 2020a). 


\section{FIGURA 2: VALOR DE LAS TRANSACCIONES MUNDIALES FINTECH POR SEGMENTOS (EN MILES DE MILLONES DE DÓLARES)}

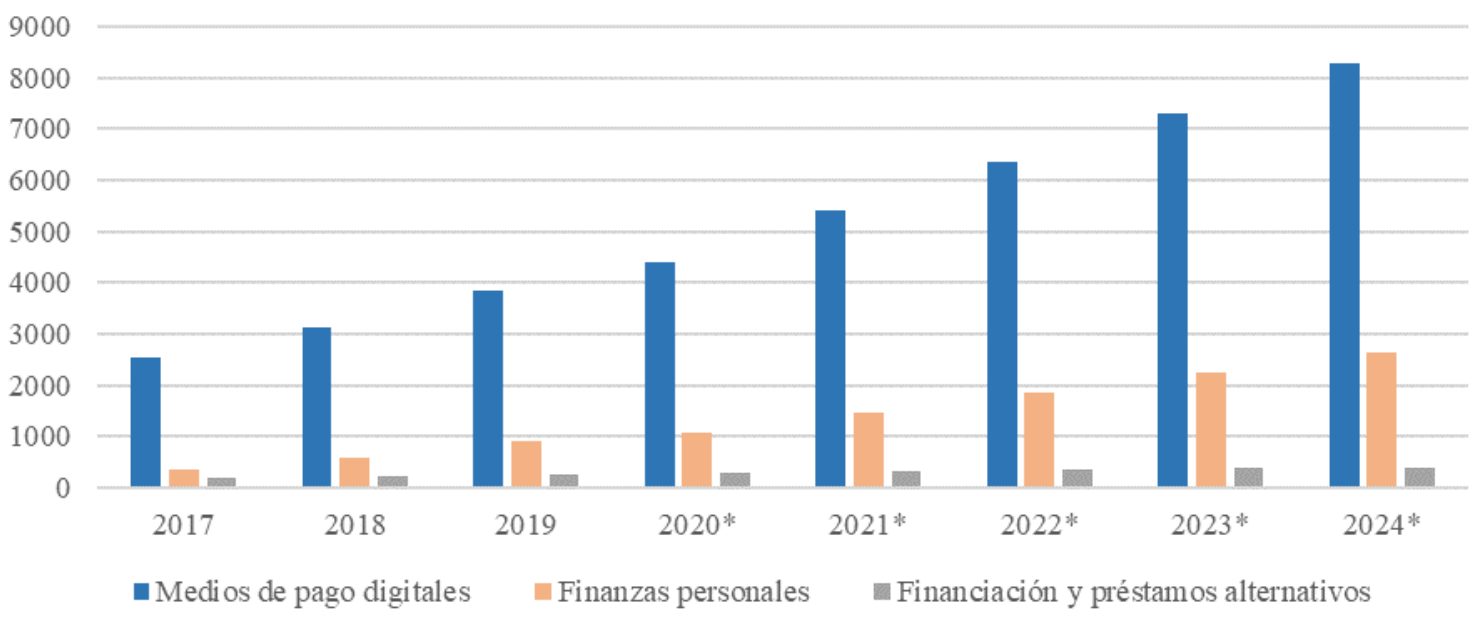

Obs.: El segmento de medios de pago digitales incluye pagos móviles y comercio electrónico; finanzas personales comprende robo-advisors y servicios automatizados de inversión; y financiación y préstamos alternativos representa crowdfunding y plataformas de préstamos peer-to-peer. Los años con asterisco recogen previsiones, las cuales están ajustadas por el impacto esperado de la COVID-19 a fecha de junio de 2020.

Fuente: Elaboración propia a partir de Fintech report 2020 (Statista, 2020a).

A esta preeminencia a nivel cuantitativo se une la indudable importancia que tienen los medios de pago en el sistema financiero, ya que estos permiten llevar a cabo una de sus funciones básicas, que es la realización de pagos. Es por ello que estas innovaciones pueden ser consideradas, dentro del fenómeno fintech, como uno de los elementos más paradigmáticos (CNMC, 2018).

Este trabajo se centra en este conjunto de innovaciones en los medios de pago, al objeto de presentar aquellas que están teniendo una mayor repercusión, explicando su funcionamiento, los principales operadores y su evolución. Tras esta exposición, se examina qué le depara el futuro a este sector, considerando las principales tendencias y las innovaciones que tendrán mayor impacto en los próximos años.

\section{INNOVACIONES EN LOS MEDIOS DE PAGO}

No resulta extraño que los medios de pago estén en el centro del fenómeno fintech teniendo en cuenta el atractivo que presenta este segmento del sistema financiero. Como se muestra en la figura 3, los ingresos por servicios de pago han aumentado a una tasa media anual del 6.8 por cien a nivel mundial desde 2010, alcanzando 1,27 billones dólares en 2017. Las proyecciones para los próximos diez años siguen siendo favorables y se prevé que los pagos generarán más de 1 billón de dólares en nuevos ingresos durante la próxima década, alcanzando los 2,42 billones de dólares en 2027. El mayor crecimiento se registrará en las economías emergentes, que impulsarán el 70 por cien del crecimiento. El acelerado crecimiento de estos países está relacionado con el menor desarrollo del sector financiero, que trae aparejado una mayor tasa de penetración de los medios de pagos digitales en estos países. 


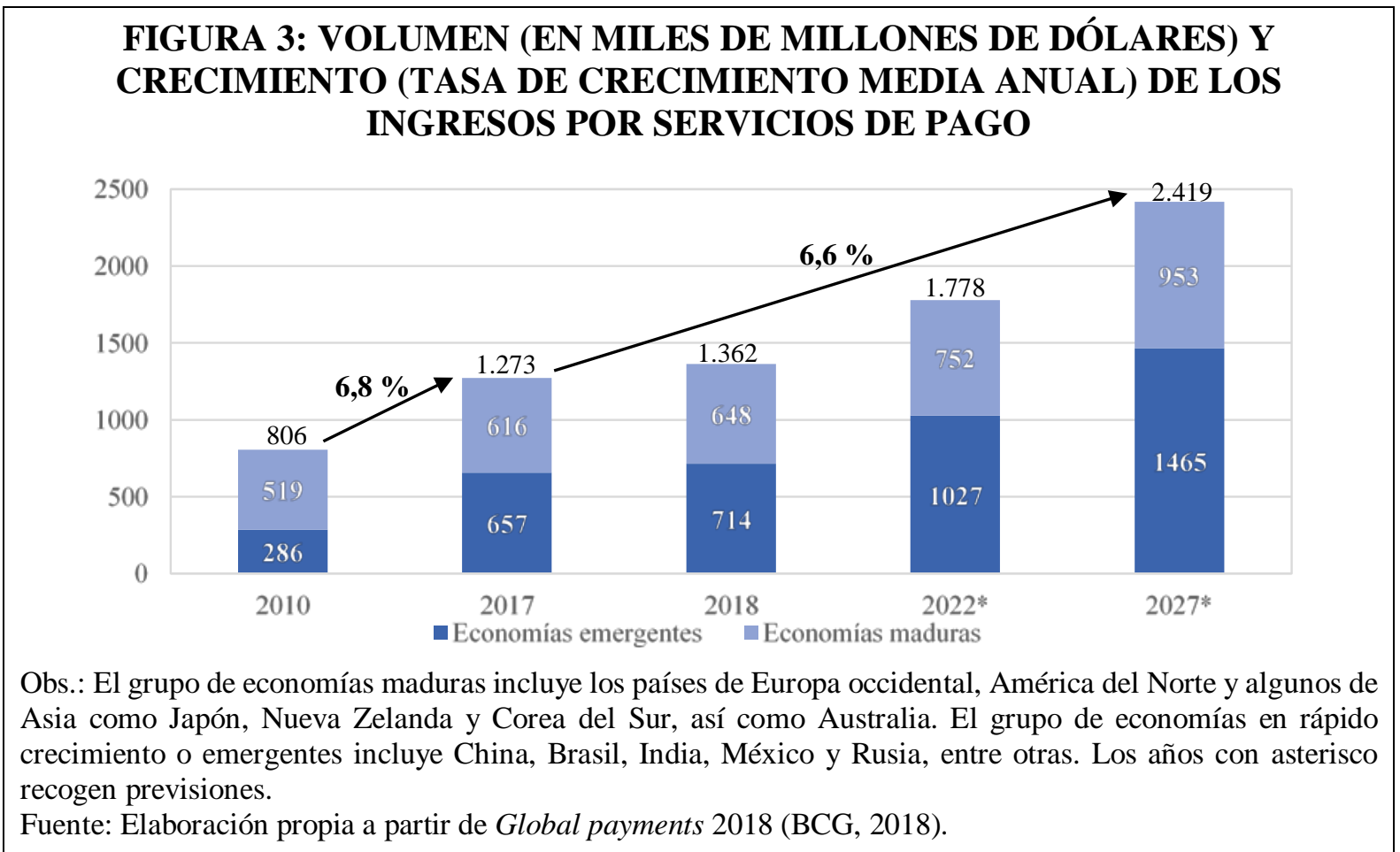

El uso de los pagos sin mediar efectivo está en aumento, impulsado por la tendencia de los pagos contactless y la disponibilidad de métodos de pago digitales, de forma que muchos países están convirtiéndose en economías con cada vez menos mediación de efectivo. Los volúmenes de transacciones no monetarias globales crecieron un 10,1 por cien entre 2015 y 2016 alcanzando los 482,6 mil millones de dólares, siendo dos regiones fundamentalmente las que impulsaron este aumento: los países emergentes de Asia representando un 25,2 por cien del crecimiento y Europa Central, Oriente Medio y África (CEMEA) con un 17,1 por cien. Se estima que en el período de 2016 a 2021, el crecimiento medio anual de las transacciones no monetarias a nivel mundial será del 12,7 por cien (Capgemini, 2018). A medida que la familiaridad con los métodos sin efectivo crece globalmente, se espera que los consumidores utilicen cada vez más medios electrónicos para pagar transacciones más pequeñas dominadas por ahora por las monedas y el efectivo. Para el 2027, se espera que, en promedio, el valor de las transacciones sin efectivo realizadas por los usuarios se reduzca de 97 dólares en 2017 a aproximadamente 54 dólares (BCG, 2018).

La revolución tecnológica en los medios de pago abre el abanico de alternativas para ejecutar pagos sin emplear efectivo, surgiendo métodos innovadores y mejorando e incorporando novedades en los ya existentes. Entre los factores que suscitan estos cambios podemos distinguir, en primer lugar, factores tecnológicos, como el creciente uso de la tecnología móvil, la cual ha provocado una demanda de nuevos productos y servicios que encajen con los nuevos estilos de vida en los que cada vez más la tecnología y los smartphones forman parte de nuestro día a día, junto con grandes innovaciones tecnológicas como el cloud computing, el big data, las tecnologías de registro distribuido (DLT), el internet de las cosas o la tecnología Near Field Communication (NFC). Todo esto permite el surgimiento de nuevos productos y servicios, así como la reducción de los costes de las transacciones, una mayor rapidez para efectuar pagos y servicios más personalizados. Por otro lado, las condiciones macroeconómicas también están contribuyendo a las innovaciones en los medios de pago puesto que, ante un entorno de bajos tipos de interés, las entidades financieras ven presionados sus ingresos a la baja y una forma de paliar esta situación es mediante la reducción de costes a través del uso de la tecnología y la innovación. Otro factor de cambio es la regulación que, si 
bien varía en función de los países, muestra una tendencia general consistente en la adaptación de las normativas a las innovaciones que van surgiendo y el fomento de nuevos medios de pago a través de las mismas. Por último, es necesario destacar la importancia de los aspectos sociales como motor de cambio en el sector ${ }^{4}$, ya que las nuevas generaciones están cada vez más familiarizadas con el uso de la tecnología y son más exigentes, buscan servicios inmediatos y tienen preferencia por la comodidad y la interoperabilidad; de hecho, numerosas encuestas muestran que es mucho más probable que los millennials utilicen servicios de pago y proveedores de servicios financieros no tradicionales (CNMC, 2018; Schindler, 2017).

Estos factores, además de suscitar cambios, atraen a nuevos competidores, tanto pequeñas startups como de mayor tamaño, las conocidas como Big Techs (entre las que se cuentan las GAFA: Google, Amazon, Facebook y Apple, pero también sus alternativas asiáticas, como por ejemplo Alibaba o Tencent). Estas grandes empresas, que están respaldadas por plataformas tecnológicas con la capacidad de proporcionar una excelente experiencia de usuario, han comenzado a capturar cuota en el mercado de los pagos a los operadores tradicionales obligándolos a ampliar, innovar, y mejorar sus ofertas ${ }^{5}$.

Las novedades en el segmento de los pagos comprenden desde innovaciones en los soportes y la infraestructura que se vienen utilizando años atrás (transferencias, tarjetas, TPV) hasta nuevos servicios que facilitan las transacciones y suponen un mayor valor añadido a los pagadores y nuevos soportes para realizar pagos totalmente nuevos y distintos a los tradicionales. El uso de algunos de los métodos que se presentan a continuación no se encuentra muy generalizado, pero se espera que en cuestión de unos pocos años formen parte de nuestro día a día (Tramazaygues, 2016).

\subsection{Monederos digitales (digital wallets)}

Uno de los nuevos soportes para los usuarios que está creciendo notablemente en los últimos años son los monederos digitales. Estos permiten realizar transacciones financieras a través de dispositivos electrónicos, ya sean tabletas, ordenadores o teléfonos móviles, almacenando información útil para realizar los pagos, como datos de las tarjetas de crédito y/o débito e información sobre cuentas bancarias de los pagadores.

\footnotetext{
${ }^{4}$ En este ámbito cabe también destacar el efecto que ha tenido la pandemia generada por la COVID-19 que, si bien ha afectado negativamente a corto plazo al conjunto del sector fintech debido a la incertidumbre y las dificultades de acceso a fuentes de capital, genera nuevas oportunidades y aumenta su potencial de crecimiento, especialmente en el ámbito de los servicios financieros digitales y el comercio electrónico (Deloitte, 2020a). Así, en respuesta a la situación creada por la COVID-19, algunos operadores fintech han reducido los costes de sus servicios, los han suspendido temporalmente, ofrecen acceso gratuito temporalmente a sus servicios o han habilitado canales para realizar donaciones a los afectados por la pandemia. Paralelamente, el aumento del comercio electrónico y el internet de las cosas debido al distanciamiento social y también la recomendación de evitar manejar dinero u otros elementos cuyas superficies pudieran infectarse tienen una influencia directa sobre el crecimiento de los medios de pago digitales.

${ }^{5}$ La oferta de servicios financieros de las GAFA no se limita sólo al ámbito de los pagos. De hecho, Apple lanzó en agosto de 2019 en EE.UU., en colaboración con Goldman Sachs, su tarjeta de crédito Apple Card, basada en la “simplicidad, transparencia y privacidad”, sin tarifas, con menores intereses y un programa de recompensas y que funciona a través de su monedero digital Apple Play. En 2011, Amazon lanzó su programa Amazon Lending, por el que concede préstamos de hasta un año a pequeñas empresas que venden a través de Amazon, y que pretende relanzar asociándose con entidades financieras. Facebook, por su parte, está en el proceso, no exento de dificultades, de crear su criptomoneda Libra. Y Google ha anunciado en noviembre de 2019 que pretende ofrecer cuentas corrientes a partir de 2020, contando con el apoyo de Citigroup. Estos ejemplos, además, muestran que al menos una parte de las iniciativas se realizan en cooperación con las entidades financieras tradicionales. En este sentido, en Observatorio de la Digitalización Financiera (2018) se recoge que sólo un 20 por cien de las empresas fintech existentes en España compiten directamente con la banca, mientras que un 32 por cien colaboran con ella y un 48 por cien juegan un papel complementario.
} 
El crecimiento del uso de estos monederos está relacionado con la importancia que ha ganado el comercio electrónico, o e-commerce, puesto que a la hora de realizar compras online la principal vía para realizar los pagos es introducir los datos de las tarjetas o los digital wallets, que a su vez están vinculados y tienen acceso a la tarjeta o cuenta bancaria del cliente. En la figura 4 se observa cómo las cifras mundiales tanto de ventas del comercio electrónico como de usuarios son muy favorables y se espera que el volumen de ventas se incremente en un 124 por cien entre 2017 y 2024 y que el número de usuarios supere los 4.600 millones en 2024, lo que supondría un gran impulso para estas nuevas alternativas de pago.

\section{FIGURA 4: VENTAS MUNDIALES DEL COMERCIO ELECTRÓNICO (EN MILES DE MILLONES DE DÓLARES) Y NÚMERO USUARIOS QUE HAN REALIZADO AL MENOS UNA COMPRA AL AÑO POR CUALQUIER CANAL DIGITAL (EN MILLONES)}

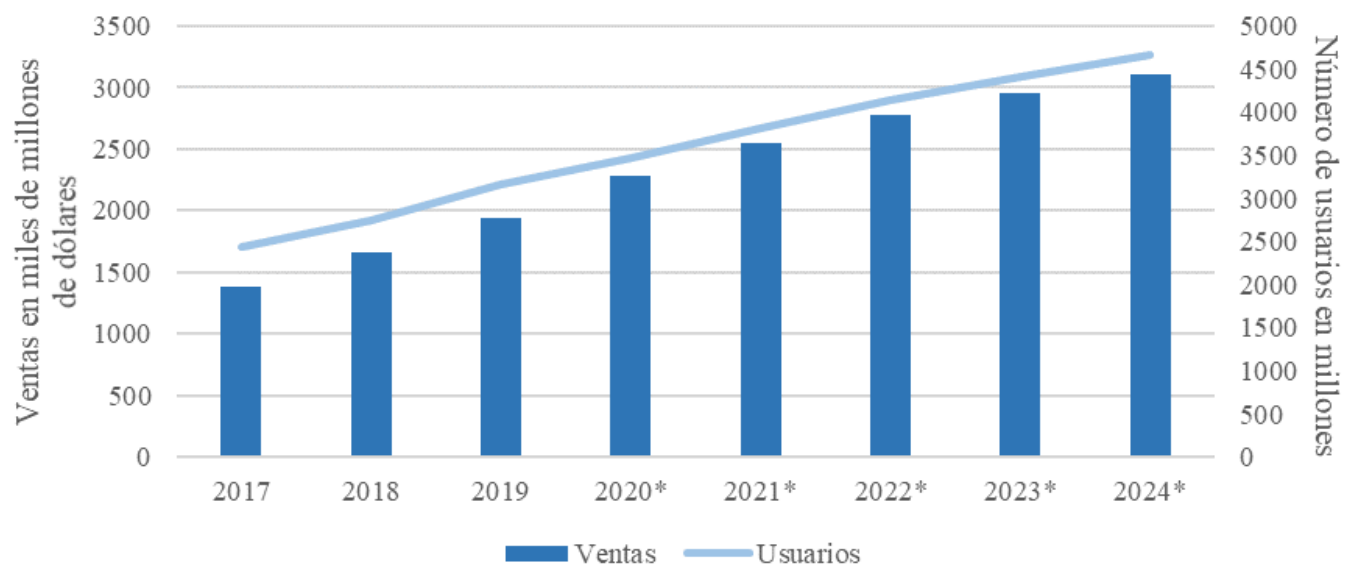

Obs.: Las cifras recogen las ventas de bienes físicos a través de canales digitales a consumidores finales (B2C), lo que excluye la venta de servicios (como billetes de avión o entradas a eventos), medios digitales (por ejemplo, descargas de música, libros electrónicos), transacciones B2B (por ejemplo, Alibaba), la reventa de bienes usados (reCommerce) o las ventas entre particulares. Los años con asterisco recogen previsiones, las cuales están ajustadas por el impacto esperado de la COVID-19 a fecha de junio de 2020.

Fuente: Elaboración propia a partir de eCommerce report 2020 (Statista, 2020b).

Dentro de los monederos digitales se puede hacer una distinción entre los monederos online (online wallets) y los monederos móviles (mobile wallets). Por un lado, los monederos online permiten realizar pagos a través de dispositivos electrónicos a distancia sin que se precise de una interacción física. A la hora de realizar una operación, estos monederos suelen interactuar con la página web en la que se realiza el pago de forma que consiguen una mayor información sobre las transacciones (CNMC, 2018). Por otro lado, los monederos móviles consisten en el uso de un dispositivo móvil para iniciar, realizar y/o confirmar cualquier tipo de compra, permitiendo así realizar cualquier transacción financiera con un smartphone y garantizando el procesamiento de los pagos de manera adecuada y segura (Hill, 2018). Fundamentalmente estos pagos operan con una tecnología electromagnética sin contacto conocida como NFC, la cual ya se encuentra incorporada en la mayoría de los smartphones, aunque también se emplean códigos QR (Quick Response) o Bluetooth (Guersent, 2016). Para efectuar un pago, el usuario debe contar con una aplicación wallet en su teléfono móvil, en la que tenga introducidos los datos de sus tarjetas, seleccionar con la que quiera realizar el pago y que el TPV del establecimiento esté equipado con tecnología NFC, y el pago se realizaría simplemente acercando el teléfono móvil a la terminal. 
Así como se ha mencionado al e-commerce como factor clave en el impulso de estos medios de pago, los teléfonos móviles y las nuevas tecnologías asociadas a los TPV también desempeñan un papel crucial gracias a su rápido desarrollo. Algunos ejemplos de TPVs de última generación serían Poynt, Clover o los TPV de Square que, además de incluir NFC, Bluetooth y códigos QR, ofrecen servicios de valor añadido como almacenamiento de información sobre las transacciones (Tramazaygues, 2016). Actualmente y de cara al futuro, el establecimiento de estos soportes es indiscutible. Este cambio ya lo adelantaba Tramazaygues (2016, p. 83): "Se espera que para 2018 dos de cada tres smartphones sean compatibles con la tecnología NFC y que para 2020 el 100 por 100 de los TPV admita esta tecnología como forma de pago".

Adicionalmente, el gran crecimiento de este sector está suponiendo una oportunidad de negocio con un gran atractivo para nuevos competidores como los Third Party Providers (TPP) o terceros proveedores de pagos como las Big Techs (Capgemini, 2018). Empresas como Google, Amazon o Apple se están adentrando en el sector incorporando estas tecnologías y creando aplicaciones, de forma que consiguen obtener una vía alternativa de ingresos (BIS, 2018; Guersent, 2016).

Así pues, los monederos digitales son ofrecidos por grandes empresas tecnológicas, sistemas operativos de móviles y por instituciones financieras, y entre las más populares cabe destacar PayPal, Apple Pay, Google Pay, AliPay y Samsumg Pay (Hill, 2018).

De esta lista, PayPal es probablemente uno de los medios más exitosos en los países occidentales, ofreciendo aplicaciones y procesos de pago simples para los consumidores y ampliamente aceptados por muchas empresas a nivel mundial. Por ejemplo, ofrece pagos electrónicos en tiempo real y transfronterizos en muchos países. PayPal permite realizar compras online y transacciones en TPVs en aquellos establecimientos que esté aceptado como medio de pago y también ofrece hacer pagos entre particulares (transferencias peer to peer, que se comentarán más adelante). Los principales beneficios de este medio de pago son la posibilidad de realizar pagos instantáneos, los costes, ya que es gratuito para transferencias nacionales y también para parte de las internacionales (sin contar el cambio de divisa, que implicaría una comisión entre 3 por cien y 4 por cien), y la seguridad, pues esta empresa garantiza que los pagos se realicen de forma fiable y los datos de la tarjeta o la cuenta bancaria introducidos en la cuenta de PayPal no se facilitan a las empresas en las que se realicen las compras; sólo con introducir el usuario de PayPal y la contraseña bastaría, de forma que el proceso de pago se simplifica y es más seguro (Guersent, 2016).

Otra gran plataforma de servicios de pagos digitales, pero de origen chino, es Alipay. Es una filial del gigante de comercio electrónico Ant Financial, aporta una solución de pagos completa incluyendo pagos en tienda, online y móviles y opera tanto en el mercado asiático como en occidente. Es la principal herramienta de pagos móviles en China y tiene la capacidad de procesar 200.000 transacciones por segundo. Su participación en el mercado chino en 2015, siendo el más grande del mundo, era del 71 por cien, pero se redujo en 2016 al 54 por cien por el crecimiento de su rival Tencent con WeChat Pay. Esta última, además de poder realizar pagos, integra en una única aplicación funciones similares a las de aplicaciones como Whatsapp, Facebook y Skype, por lo que consiguió un gran impacto alcanzando una cuota del 40 por cien en dicho mercado. En España, Alipay ha llegado a acuerdos con entidades como BBVA, Caixabank y Banco Sabadell para permitir que se realicen pagos en establecimientos que cuenten con TPVs de estas entidades.

También hay que destacar en este sector las plataformas de Samsung Pay, Apple Pay, Google Pay (la cual integra las anteriores aplicaciones de Google Wallet y Android Pay en una única aplicación) y Amazon Pay. Apple y Samsung Pay sólo permiten realizar pagos con dispositivos de sus marcas y Google Pay a través de todos los dispositivos móviles 
Android, ordenadores, iPhone y iPad. Además, Google incluye como métodos de pago varias opciones que conllevan la vinculación de tarjetas de crédito/débito, de la cuenta bancaria (esta opción sólo está disponible en EE.UU. por el momento) y, en el caso de pagos móviles, también la cuenta de PayPal (Google Pay, 2019). Estos métodos comparten que sólo se pueden utilizar en aquellos negocios que hayan sido aceptados como medio de pago y tienen en común ventajas en seguridad, y es que ninguno de ellos al realizar compras en tiendas físicas transmite información sobre el número de cuenta o de tarjeta del cliente, sino que éste se cifra y se utilizan diversos códigos y sistemas de seguridad (Hill, 2018).

La figura 5 muestra que, en la actualidad, Apple Pay es el método de pago móvil más usado en España, siendo utilizado por el 32 por cien de los usuarios de estos servicios. Le sigue la aplicación de Caixabank con un 22 por cien y, tras ella, se sitúa Google Pay para continuar, de nuevo, con la de otro banco, BBVA Wallet. Esto evidencia que los bancos españoles están desarrollando soluciones para adaptar su oferta a la nueva era digital y se preocupan por ofrecer más servicios innovadores y de mayor valor añadido a sus clientes, haciéndose de este modo hueco en el ecosistema fintech.

\section{FIGURA 5: PREFERENCIA POR DISTINTOS SERVICIOS DE PAGO MÓVILES EN ESPAÑA (PORCENTAJE DE USUARIOS QUE HAN EMPLEADO CADA SERVICIO EN LOS ÚLTIMOS 12 MESES EN COMERCIOS, RESTAURANTES U OTROS PUNTOS DE VENTA)}

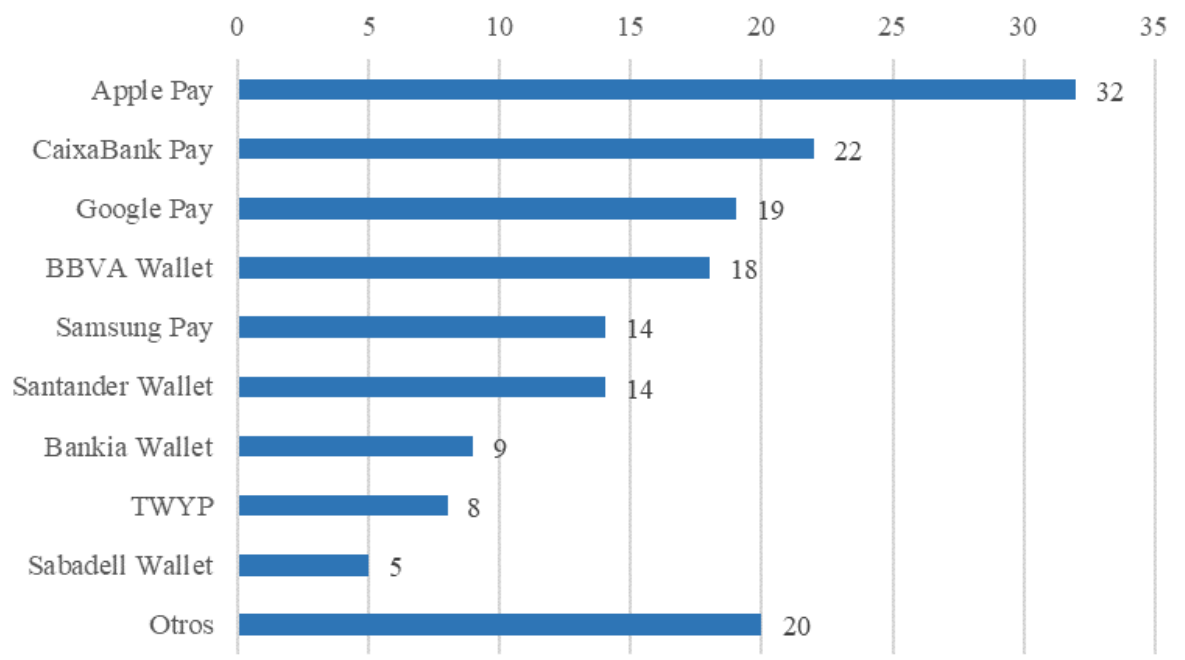

Obs.: Las encuestas se realizaron en dos periodos: del 5 de agosto al 20 de septiembre de 2019 y del 13 de febrero al 27 de marzo de 2020. Los encuestados podían seleccionar varios servicios, por lo que la suma de los porcentajes supera el 100 por cien.

Fuente: Elaboración propia a partir de Global consumer survey 2020 (Statista, 2020c).

En definitiva, los monederos digitales representan una versión digitalizada de las tarjetas de crédito $\mathrm{y}$, a diferencia del uso de las tarjetas tradicionales, ofrecen ventajas como la comodidad, puesto que estas plataformas almacenan los datos en las aplicaciones o en sus webs evitando así tener que introducir los datos bancarios en cada transacción que se realice y permiten realizar pagos desde cualquier lugar independientemente de la ubicación del pagador y del establecimiento al que se le abona el dinero. Otro punto fuerte es la personalización de los servicios, ya que estos soportes almacenan información sobre las transacciones para ofrecer un mayor valor añadido y rapidez (Tramazaygues, 2016). Por todo lo mencionado, no sería de extrañar y es previsible que los métodos de pago clásicos como el efectivo y las tarjetas se vean 
superados, en cuestión de unos años, por estos nuevos medios basados en monederos y dispositivos online y móviles (Deloitte, 2016).

\subsection{Transferencias P2P (peer to peer)}

Otro modelo de negocio importante en el sector de los pagos son los servicios de pago P2P. Las transferencias de dinero P2P consisten en transferencias de dinero entre particulares realizadas a través de Internet, por medio de dispositivos como ordenadores o teléfonos móviles, y que pueden ser bien a nivel nacional o internacional (Statista, 2020a). Para efectuarlas es necesario previamente vincular una tarjeta o la cuenta bancaria a una aplicación, aunque algunas permiten vincular la cuenta de PayPal como es el caso de Facebook o Google. Una vez vinculada, realizar una transferencia de dinero a otra persona supone un proceso simple y rápido, similar a enviar un mensaje: simplemente se selecciona al beneficiario a través de su nombre de usuario, añadiendo su correo electrónico o por su número de teléfono (en función de la plataforma que se use), se comprueba que se trata de la persona adecuada y se efectúa la transferencia. La gran ventaja de este procedimiento es que sustituye muchas transacciones que, de no ser así, se realizarían en efectivo, cheque o tarjeta, es decir, consiguen reemplazar al efectivo en transacciones en donde resultaba difícil sustituirlo ofreciendo una alternativa más rápida y segura (Guersent, 2016).

El sector de los pagos entre particulares resulta de gran atractivo. En 2018 se realizaron a nivel mundial transacciones por valor de 66.000 millones de dólares y en 2023 se espera que ese valor se eleve a más del doble, 147.000 millones de dólares (Statista, 2019a). Es por esto que para muchos monederos digitales como los que se han analizado anteriormente, los pagos peer to peer son considerados como una buena forma de ampliar su cartera de servicios y, por tanto, como una oportunidad para atraer a más clientes. Por ejemplo, Alipay y WeChat Pay, aprovechando la gran penetración y la base de usuarios que tienen, se han convertido en líderes de los pagos P2P en China. Google Pay y Apple Pay también permiten realizar este tipo de pagos entre particulares, pero por el momento sólo en algunos países (Capgemini, 2018; Google Pay, 2019). PayPal también permite realizar pagos entre particulares a nivel nacional e internacional, puesto que opera en gran parte del mundo; además, cuenta con Venmo, una empresa de servicios de pago entre particulares que opera sólo en EE.UU. Venmo tiene un diseño similar al de una red social y permite sincronizar los amigos de Facebook o la lista de contactos del teléfono móvil con la aplicación para realizar transferencias con estas personas. Su rival principal en EE.UU. es Square Cash, ahora denominada Cash App, que fue desarrollada por Square, empresa de servicios financieros estadounidense cuya principal actividad se basa en fabricar lectores de tarjetas y todo tipo de soportes (tanto hardware como software) que permitan a las empresas poder recibir y registrar los pagos de sus clientes (Hill, 2018).

Los bancos también se han sumado al sector de los pagos P2P reaccionando frente a la oferta de estas empresas para tratar de impedir que los usuarios opten por estas nuevas aplicaciones, mejorar sus servicios haciéndolos más rápidos, con menos comisiones y adaptándolos a los avances tecnológicos. En EE.UU., ante el éxito de Venmo, PayPal y Square Cash, un consorcio de treinta bancos estadounidenses se unió en 2017 para crear Zelle, una aplicación con un funcionamiento muy similar al de Venmo que permite realizar transferencias de fondos entre usuarios en cuestión de minutos (Hill, 2018). Como se muestra en la figura 6, el volumen de pagos móviles P2P realizados a través de Venmo, Square y Zelle en el último trimestre de 2019 alcanzó los 114.000 millones de dólares; para los años posteriores, las predicciones del número de usuarios aumentarían en las tres compañías, pero en mayor medida en Zelle, que es la que muestra la tendencia más ascendente. 


\section{FIGURA 6: VOLUMEN DE PAGOS MÓVILES P2P A NIVEL MUNDIAL DE VENMO, SQUARE Y ZELLE (EN MILES DE MILLONES DE DÓLARES)}

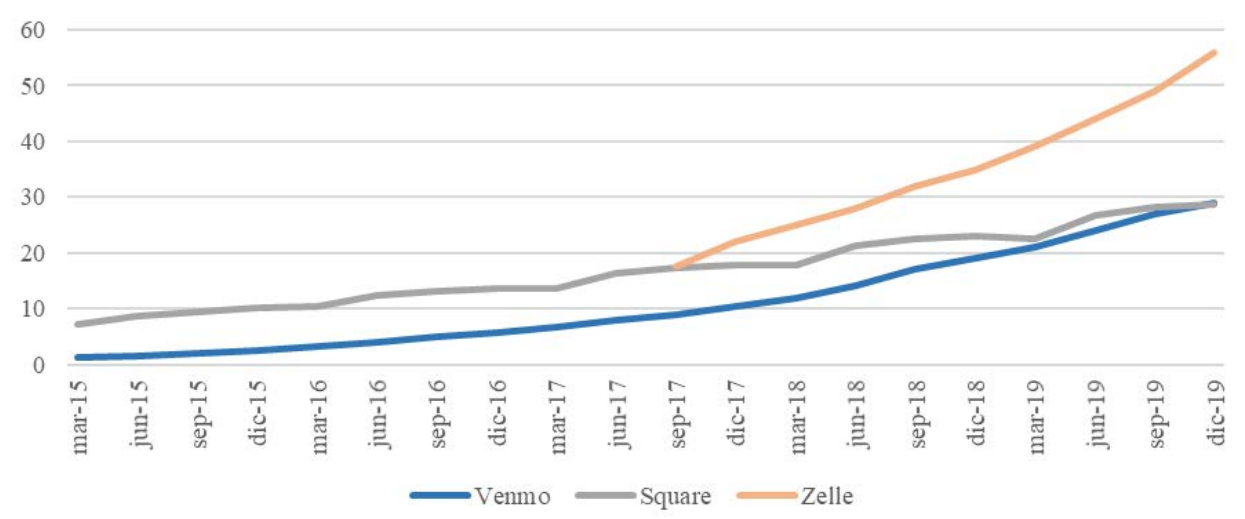

Obs: Square está disponible en EE.UU., Canadá, Japón, Australia y Reino Unido. Venmo y Zelle sólo en EE.UU.

Fuente: Elaboración propia a partir de PayPal (2020), Ritcher (2017), Square (2020) y Zelle (2020).

Las principales entidades financieras españolas también lanzaron su alternativa en cuanto a pagos instantáneos entre particulares uniendo fuerzas bajo la aplicación Bizum (Capgemini, 2018). Actualmente cuenta con la participación de sesenta y seis bancos, tiene más de nueve millones de usuarios y a través de la aplicación se realizan más de 135 millones de transacciones. Inicialmente sólo permitía realizar pagos P2P de forma rápida, fácil, segura y gratuita conociendo el número de móvil de la contraparte, pero con el tiempo ha ido incorporando más funciones como la posibilidad de contribuir a proyectos de ONGs y asociaciones relacionadas con esta plataforma, más recientemente, el poder realizar compras online en los establecimientos que lo acepten como medio de pago y, de cara al futuro, se está trabajando para que permita realizar pagos en establecimientos físicos como un monedero móvil (Bizum, 2020).

A diferencia de Bizum, Twyp es una plataforma desarrollada únicamente por el banco ING, aunque puede ser utilizada por cualquier usuario sin importar del banco que sean. Twyp incluye, aparte de transferencias entre iguales, servicios que Bizum aún no tiene disponibles como la retirada de efectivo o pagos en comercios físicos que operan con este medio mediante la aplicación de Twyp. Es más, con Twyp es posible pagar en cualquier comercio que disponga de la opción de pago móvil añadiendo la tarjeta Twyp a Google Pay o Apple Pay, es decir, que permite generar una tarjeta de prepago virtual que funciona con los monederos móviles (Twyp, 2020).

Dado el gran atractivo del sector, las redes sociales también se han subido al carro de la innovación en pagos entre iguales. Por ejemplo, la aplicación de mensajería WhatsApp de Facebook está probando un servicio de pagos en la India. Eligió la India debido al gran incentivo a estos medios de pago otorgado por el gobierno del país que, en 2016, inició una política de desmonetización fomentando las transacciones no monetarias y porque la región representa un motor importante en el crecimiento de las transacciones no monetarias a nivel mundial (Capgemini, 2018). A partir de abril de 2019 también comenzó a hacer pruebas en México en colaboración con Banco Santander México. Este nuevo servicio a través de la aplicación de Whatsapp permitirá a los usuarios realizar transferencias de dinero entre particulares simplemente seleccionando a los contactos de la red social a los que se quiere enviar el dinero (Sánchez, 2019).

Otro caso de pagos P2P a través de redes sociales es el de Facebook, que mediante su aplicación Messenger permite llevar a cabo transferencias de dinero a los contactos conectando 
una tarjeta bancaria, pero estos pagos sólo se pueden realizar siempre que los bancos del pagador y el beneficiario se encuentren dentro del mismo país y operen con la misma divisa (Facebook, 2018).

En cuanto a las transferencias internacionales, existen empresas fintech que proporcionan pagos internacionales como Transferwise, Xoom, PayPal o WorldRemit, que están amenazando la participación que tienen los bancos y otras empresas veteranas en la prestación de servicios de pago como Western Union y MoneyGram. Cuando se trata de transferencias de dinero transfronterizas, el atractivo depende en gran medida del coste. La propuesta de valor de las fintechs está basada en facilitar la ejecución de las transacciones a través de un proceso íntegramente online, proporcionar servicios más ágiles que permitan la transferencia de dinero a cualquier parte del mundo en cuestión de unos minutos, saber en cada instante dónde se encuentra el dinero pudiendo seguir la trayectoria de la transacción, la transparencia y con tarifas de precios más bajos y competitivos que las entidades tradicionales (Tramazaygues, 2016).

Aunque, como se ha visto, son numerosas las innovaciones en las transferencias P2P, el desafío en última instancia para las plataformas fintech es garantizar la seguridad y la confianza en las transacciones mediante procesos de identificación, encriptación, y detección de fraude.

\subsection{Pagos en factura telefónica}

Los pagos en factura telefónica también conocidos como "direct carrier billing" son un medio de pago online que permite a los usuarios cargar los pagos que realicen en la factura mensual de su teléfono móvil (Funcas y KMPG, 2018). Existen diferentes segmentos que utilizan este medio de pago como juegos, redes sociales como Facebook, plataformas de contenido multimedia tales como Netflix o Spotify, y tiendas de aplicaciones en el móvil App Store o Play Store, entre otros. El proceso para realizar los pagos es simple, como se muestra en la figura 7. A la hora de realizar la compra de un producto se tendrá que introducir el número de teléfono del usuario (una vez ya hecho para una compra no se tendrá que volver a cumplimentar para el resto), al realizar una solicitud de compra el cliente recibe un código PIN a través de un SMS para confirmar la compra y el usuario realiza el pago en su factura mensual de móvil (Fortumo, 2019).

\section{FIGURA 7: PROCESO DE PAGO MEDIANTE CARRIER BILLING}

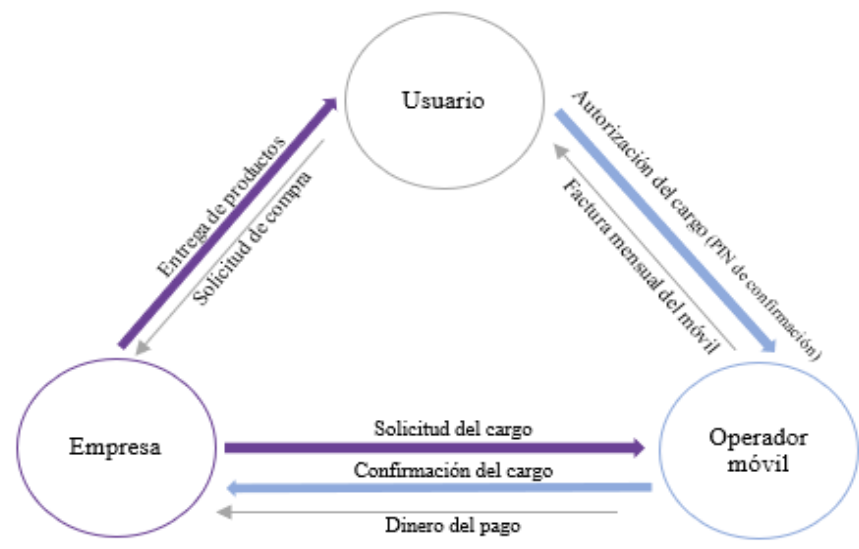

Nota: La figura ilustra los diferentes procesos que se llevan a cabo a la hora de realizar pagos mediante factura telefónica distinguiendo entre los tres actores que intervienen en la transacción: el usuario, la empresa en la que el usuario compra un producto y el operador móvil a través del que se efectúa el pago.

Fuente: Elaboración propia. 
Las empresas pueden encontrar ventajas en el carrier billing por el alcance, la sencillez y la seguridad. Esto es, este medio de pago es online y se realiza por medio de un smartphone y, por tanto, puede llegar a muchos más usuarios que otro medio como la tarjeta, ya que hay 3.500 millones de usuarios de Internet en el mundo y el 40 por cien no cuenta con tarjeta de crédito. Por la sencillez, pues se basa en un flujo de pago muy simple. Y por seguridad, ya que no es necesario enviar información personal para realizar la transacción (Fortumo, 2019). Este método está teniendo un mayor éxito en países emergentes que en países desarrollados dado el gran peso en estos últimos del sector bancario. En los países emergentes, principalmente en la zona del Pacífico de Asia, Europa del Este, África y Latinoamérica, se produjo un gran cambio con la incorporación de estos países al mundo online y con la introducción de los smartphones; de hecho, la tasa de adopción es muy elevada, 8 de cada 10 personas en estas zonas posee un teléfono móvil. Por esta razón, y al ser países con poca presencia de la banca, no cuentan con una gran oferta de servicios financieros y el uso de tarjetas como medio de pago no está muy extendido, por lo que el método carrier billing está creciendo rápidamente (Funcas y KPMG, 2018).

A modo resumen y teniendo en cuenta lo comentado anteriormente en relación con las innovaciones en los medios de pago, en la figura 8 se recogen las diferentes formas de pago en la actualidad agrupadas en función de la finalidad que se persiga.

\section{FIGURA 8: TIPOS DE MEDIOS DE PAGO EN FUNCIÓN DE LA NECESIDAD}
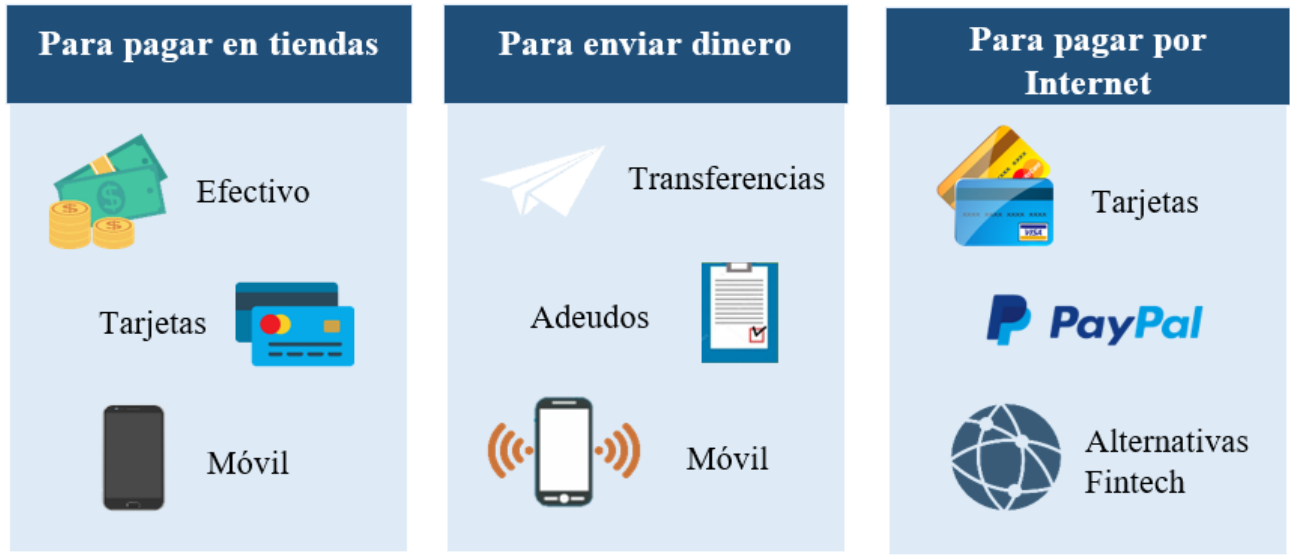

Fuente: Elaboración propia.

\section{PERSPECTIVAS DE FUTURO}

No cabe duda de que la tecnología está cambiando el sector financiero y se están desarrollando innovaciones que modifican la manera en la que se estructuran, se prestan y se utilizan los servicios financieros, incluidos los servicios de pago. En este apartado se presentan las innovaciones que, previsiblemente, tendrán mayor impacto en los próximos años, examinando qué le depara el futuro a este sector, las principales tendencias y las posibles aplicaciones a los medios y sistemas de pago.

\subsection{Tecnología DLT (distributed ledger technology)}

En primer lugar, es de destacar la tecnología blockchain y, en general, las DLT (distributed ledger technology o tecnología de registro distribuido). Una DLT es una base de datos descentralizada gestionada por múltiples usuarios, de forma que todos tienen acceso a los datos y al historial de registros, pudiendo verificar las transacciones colectivamente. Este hecho 
hace que no sea necesaria la existencia de una autoridad central o un intermediario para actualizar la base de datos o realizar una transacción (Angelov, 2019). La tecnología DLT combina redes P2P (para conectar a los diferentes usuarios o nodos), criptografía (para aportar seguridad sobre la información que se intercambia, autenticando al emisor y cifrando la información) y algoritmos de consenso (mecanismos para llegar a un consenso entre los participantes y garantizar que no haya fraudes ni duplicidades). El blockchain es un caso particular de DLT, basado en bloques que forman una cadena y el proceso que se sigue hace que cuando se realiza una transacción los registros sean inalterables, pues los registros se generan de forma automática conectándose con el resto de la cadena de bloques por orden cronológico; por tanto, ante cualquier modificación, todos los participantes observarían cómo la cadena se rompería, de ahí la gran seguridad que aporta esta tecnología y la dificultad a la hora de cometer fraudes o hackear este sistema (Romero, 2018). En la figura 9 se puede observar de forma simplificada el funcionamiento de la tecnología blockchain al realizar una transacción.

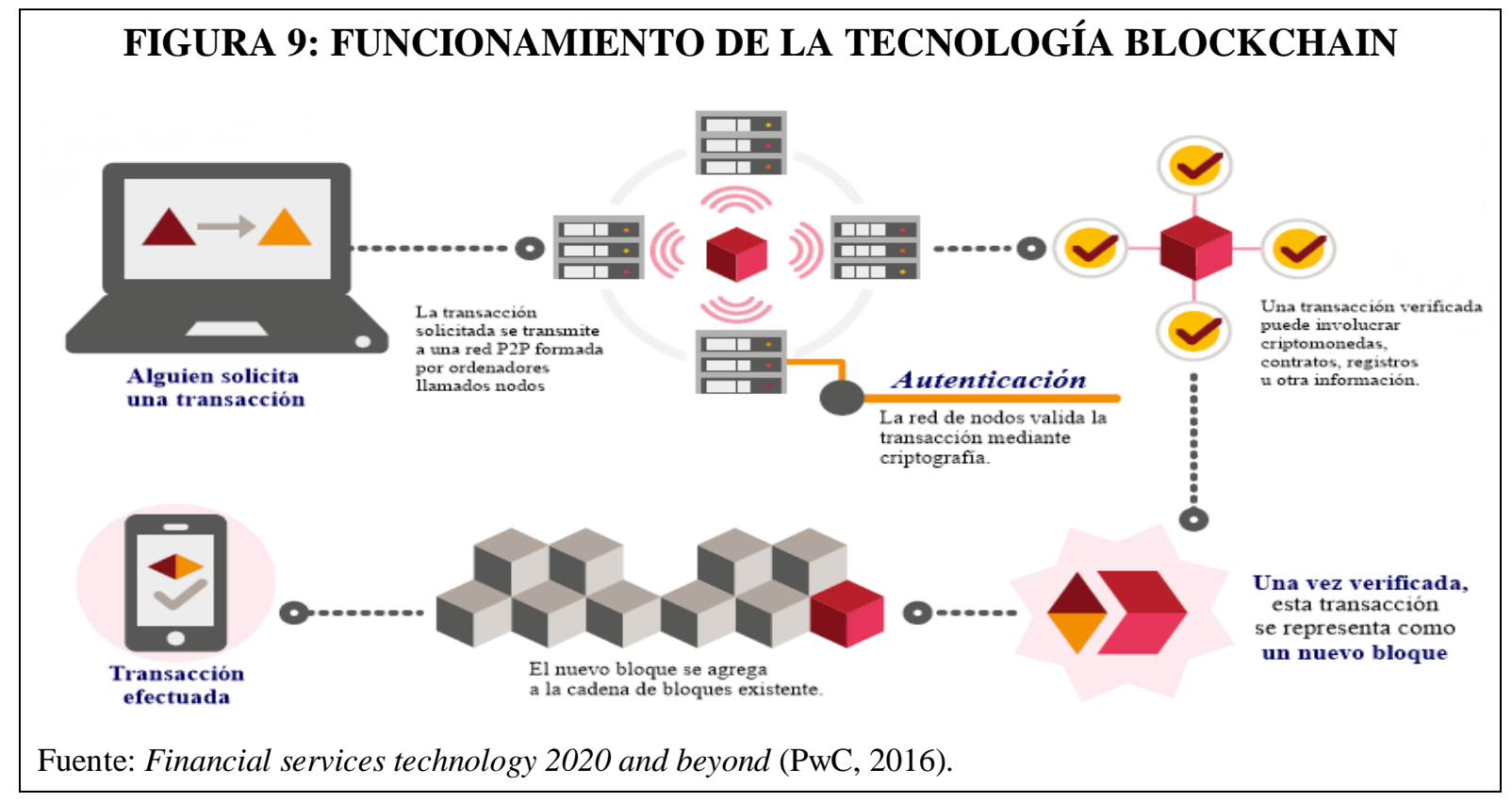

La tecnología DLT, aunque cuenta con limitaciones como la ausencia de una regulación clara y la falta de confianza por los usuarios, tiene un gran potencial de aplicación en diversos sectores como el financiero, asegurador, para trámites burocráticos e incluso en sanidad (PwC, 2016). En el sector financiero, en el corto plazo, se está investigando su uso para mejorar la experiencia del cliente mediante las funciones de integridad y seguridad de datos que ofrece.

A más largo plazo, la tecnología DLT podría emplearse en el ámbito de los pagos para mejorar su eficiencia, la gestión de la identidad digital de clientes, realizar pagos transfronterizos, transferencias P2P e incluso pagos y liquidaciones interbancarias (Capgemini, 2018). Concretamente, en el segmento de los pagos se están llevando a cabo diversos proyectos en relación con las transferencias de fondos internacionales. Entre los posibles beneficios de realizar pagos internacionales a través de DLT cabe destacar la simplificación de todo el proceso al reducir la intermediación (se eliminaría la figura de mediadores como Visa, Mastercard, cámaras y entidades de compensación), liquidaciones en tiempo real, gran reducción en los costes, una mayor transparencia, menores riesgos de fraude y una menor necesidad de liquidez (Tramazaygues, 2016). 
En España, en relación con esta tecnología, desde octubre de 2017 existe una alianza entre empresas e instituciones de distintos sectores (Banco Santander, Banco Sabadell, Repsol, Everis, Endesa, BBVA, Grant Thornton, Garrigues, entre otras), sumando en la actualidad más de 550 miembros. Esta unión, denominada Alastria, supone la primera red blockchain semipública cuyo objetivo es desarrollar innovaciones a través del uso de blockchain al amparo de la regulación española (Garijo, 2018).

Según el World Economic Forum (2016), el uso de blockchain se convertirá en una parte integral de la infraestructura operativa de las instituciones financieras y, en los próximos años, en una era de delitos cibernéticos y estrictos requisitos regulatorios, un sistema altamente resistente al fraude para proteger y autenticar casi cualquier tipo de transacción podría tener un impacto revolucionario en el sistema financiero. Todo apunta a un extraordinario incremento en los niveles de inversión de las compañías para crear productos y soluciones específicas basadas en DLT. De hecho, como se puede observar en la figura 10, en los últimos años se ha producido un notable aumento de la inversión en blockchain en las distintas zonas del mundo, destacando el papel de EE.UU., Europa occidental y China que, según las predicciones para el período de 2018 a 2022, se situarían como precursoras (KPMG, 2019).

\section{FIGURA 10: GASTO MUNDIAL POR REGIONES EN SOLUCIONES BLOCKCHAIN (EN MILES DE MILLONES DE DÓLARES)}

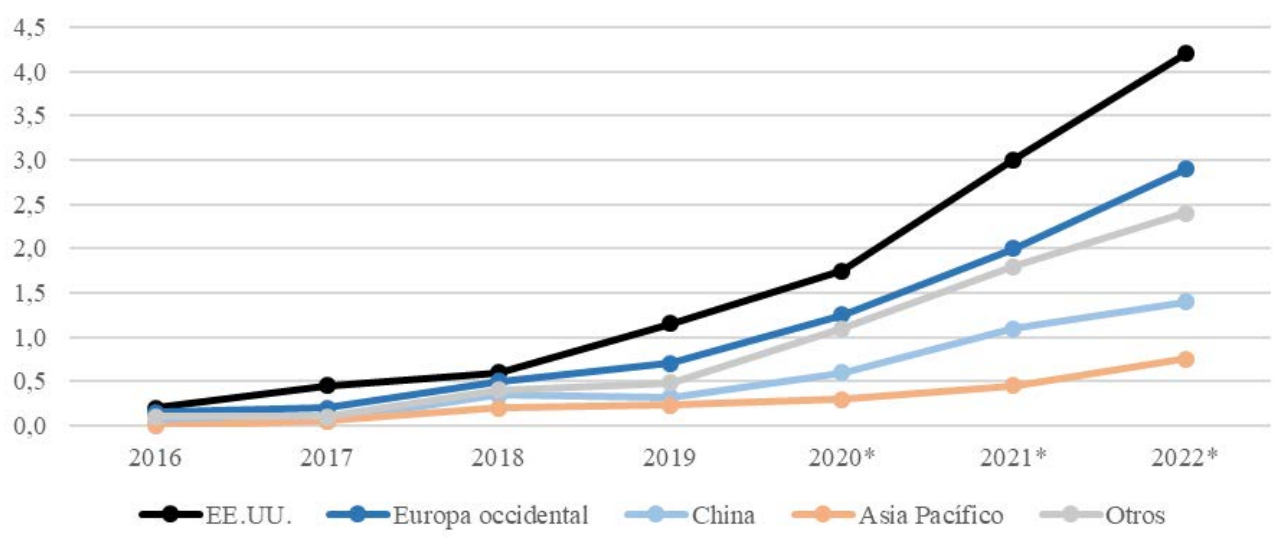

Obs.: Los años con asterisco recogen previsiones.

Fuente: Elaboración propia a partir de Statista (2019b) y Houbi Research (2020).

\subsection{Internet de las cosas y dispositivos inteligentes basados en inteligencia artificial}

Otras tecnologías que están alterando todos los esquemas del sector financiero y que influyen también en los pagos son el Internet de las cosas y los dispositivos inteligentes, basados en la inteligencia artificial, los cuales están revolucionando el sector financiero (Capgemini, 2018).

Por un lado, la inteligencia artificial (también conocida como AI por sus siglas en inglés) consiste en un sistema con una codificación que hace que un ordenador o un dispositivo esté capacitado para recordar patrones, analizar, comprender y tomar decisiones empleando una lógica parecida a la de un ser humano (BIS, 2018). La AI combina esta inteligencia con la posibilidad de automatizar tareas rutinarias y simples, suponiendo un consecuente ahorro en costes y tiempo. Además, cuenta con capacidad de análisis de grandes bases de datos (big data) y de aprendizaje, por lo que se puede emplear en tareas como estudios de mercado, recopilación de datos, gestión de carteras de activos, análisis de mercados bursátiles y servicios de asesoramiento (Angelov, 2019). 
Por otro lado, relacionado con la aplicación de la AI, está el internet de las cosas (por sus siglas en inglés IoT), el cual ha motivado la proliferación de dispositivos de nuestro día a día que cuentan con sistemas operativos y sensores que recogen información, la analizan y realizan acciones en base a ella. Hasta ahora, el crecimiento del IoT en servicios financieros se ha producido principalmente en el sector de los pagos, seguros y en la banca (PwC, 2016).

El principal exponente de la AI y del IoT son los smartphones, pero los bancos están estableciendo asociaciones con fabricantes de tecnología portátil para permitir a los clientes realizar pagos móviles utilizando wearables como son los smartwatches o las pulseras de actividad física. Un ejemplo de esto es Fitbit Pay, una pulsera de actividad física para pagar en tiendas con tecnología NFC. También es de destacar el Dash Button de Amazon, el cual permite a los clientes reponer artículos de uso cotidiano comprándolos a través del monedero digital de Amazon con un simple clic; sin embargo, este dispositivo ya no se comercializa por el auge de los asistentes como Alexa o Google Home. Estos asistentes de inteligencia artificial permiten realizar pagos por voz dando lugar a servicios de mayor valor añadido basados en las conversaciones con el asistente de voz. Asimismo, Jaguar y Shell se han asociado para desarrollar una aplicación de pagos mediante el automóvil basada en el uso del GPS sin necesidad de que medie efectivo o una tarjeta de crédito física (Capgemini, 2018). Un último ejemplo de IoT está en los electrodomésticos del hogar, como frigoríficos, televisiones, hornos, lavavajillas. Así, por ejemplo, el frigorífico Family Hub, lanzado al mercado en 2016 por Samsung con la aplicación Groceries desarrollada junto con Mastercard, hace posible realizar la compra y el pago de la misma a través del frigorífico. Además, el frigorífico, gracias al uso de AI, recuerda patrones de compra de las familias y es capaz de realizar sugerencias de compra personalizadas (Pérez, 2016). Con el aumento del IoT, hay un cambio creciente en el panorama de pagos hacia pagos iniciados por dispositivos. Además, estas tecnologías, complementadas con infraestructuras de pagos en tiempo real y el fácil acceso a la identidad de las partes involucradas en las transacciones, están creando un ecosistema cada vez más conectado entre dispositivos, sistemas e instituciones financieras (Capgemini, 2018).

No obstante, estas innovaciones cuentan con ciertos impedimentos como la falta de organización de la información en grandes bases de datos para poder procesarlas a través de AI, el miedo a que esta tecnología sustituya la mano de obra humana en algunos puestos de trabajo, ciertos riesgos asociados a tareas que no permiten que la AI pueda funcionar de manera autónoma y problemas éticos relacionados con la protección de la privacidad debido a la recopilación masiva de datos que realizan estas tecnologías (Angelov, 2019; PwC, 2016).

\subsection{Computación cuántica, biometría y tokenización}

En otro orden de cosas, la computación cuántica o “quantum computing”, a pesar de aún no estar desarrollada, también está en la vanguardia de la investigación tecnológica. Esta innovación se basa en las leyes de la mecánica cuántica y es capaz de abordar dificultades que los sistemas de computación existentes no pueden solventar. Son ordenadores con un gran potencial, totalmente distintos a los actuales y con capacidad para procesar gran variedad de datos en menos tiempo. Tiene unos beneficios enormes en el campo de la ciencia y se prevé que revolucione los sectores de la inteligencia artificial, la medicina, la investigación espacial y la ciberseguridad (Rjaibi, 2018). En relación a este último sector, se está investigando sobre la criptografía cuántica y su aplicación al sector financiero. Ésta podría suponer una forma de seguridad prácticamente imposible de hackear puesto que la física cuántica permitiría que la información se teletransporte de un sitio a otro y, en caso de ataque, la información se destruye. 
La computación cuántica utiliza qubits ${ }^{6}$, los cuales podrían formar parte, en un futuro y en el ámbito de los sistemas de pago, de la creación de dinero cuántico, e incluso tarjetas cuánticas que no podrán ser copiadas (Angelov, 2019). Aunque hay una gran variedad de posibles aplicaciones del quantum computing en las finanzas, como su uso relacionado con blockchain, criptomonedas, criptografía o la seguridad de las transacciones financieras, entre otras, es probable que para ver su implementación real tengan que pasar décadas (Rjaibi, 2018).

Un gran desafío de cara al futuro del sector financiero son los inconvenientes relativos a la seguridad de las innovaciones ya que, por ejemplo, en 2019, los hackers lograron robar más de 7.000 bitcoins de cuentas en Binace, la mayor plataforma de criptomonedas a nivel mundial, representando unos 36 millones de euros. Novedades relacionadas con la seguridad como técnicas biométricas y la tokenización pudieran ser la clave para superar estos retos (Deloitte, 2016).

La biometría es una tecnología que utiliza determinados comportamientos o elementos físicos para la identificación de las personas y la validación de pagos, es decir, permite al usuario identificarse basándose en rasgos únicos que caracterizan a las personas. Entre las técnicas biométricas podemos distinguir el reconocimiento facial (como por ejemplo el Face ID de Apple), el uso de la huella dactilar (en lectores de huellas incluidos en TPVs o el caso del ID Touch de Apple, que permite confirmar pagos contactless), el escáner del iris o de las venas de la mano o la identificación mediante el ritmo cardíaco. La biometría supone grandes ventajas de seguridad puesto que evita que las contraseñas puedan ser robadas y reduce los fraudes (Avendaño, Pérez y Queizán, 2016). Estas tecnologías ya están siendo adoptadas por muchos bancos y empresas tecnológicas y se espera que experimenten un gran crecimiento; de hecho, en 2017, en comparación con el año anterior, el uso de técnicas biométricas en pagos móviles se triplicó (Angelov, 2019).

La tokenización también incrementa la seguridad en las transacciones evitando que los datos bancarios se transmitan a los comercios en los que se realizan compras. Funciona mediante un token o un número, distinto en cada operación, que reemplaza la información bancaria del usuario. Google, Samsung y Apple Pay ya hacen uso de estas técnicas, de hecho, Apple, ya en 2014, lanzó un sistema de pago mediante tokens en el que el cliente también hacía uso de técnicas biométricas autorizando la operación con la huella dactilar (Avendaño et al., 2016).

En definitiva, las innovaciones que están por venir pueden contribuir a resolver algunos de los problemas antiguos y recientes en los pagos y abren nuevas oportunidades que hasta ahora sólo estaban recogidas en la ciencia ficción.

\section{CONCLUSIONES}

A pesar de que se apela al término fintech como si se tratase de un fenómeno muy novedoso, realmente no es así. Fintech implica la transformación, el avance del mundo financiero gracias a la tecnología, y esto viene desarrollándose desde hace décadas. No obstante, el uso del término fintech para denominar a este fenómeno sí es reciente y se vincula con los cambios más disruptivos del sector que se vienen sucediendo en los últimos años.

Este trabajo se ha centrado en las innovaciones fintech en los medios de pago, puesto que desempeñan una de las funciones básicas del sector financiero, constituyen el caso más paradigmático del fenómeno fintech y, además, los medios de pago son uno de los sectores en los que se están sucediendo los cambios más visibles desde el punto de vista del público.

\footnotetext{
${ }^{6}$ A diferencia de los bits usados en la computación tradicional que pueden tomar un valor 0 o 1, los bits cuánticos o qubits pueden tomar valores de 1 y 0 simultáneamente.
} 
Las principales tendencias de cara a los próximos años en relación a los medios de pago se pueden reflejar de forma resumida en los siguientes puntos:

- El uso del efectivo con el paso del tiempo se irá reduciendo a favor de otros medios de pago como son las tarjetas contactless, los monederos digitales y los wearables.

- Existe un auge de plataformas de pago no bancarias, como PayPal, que facilitan las transacciones P2P; no obstante, los operadores tradicionales bancarios, en respuesta a esta competencia, cuentan ya con plataformas similares en este campo como Bizum o Twyp en el caso de España.

- El fenómeno open banking o de apertura de los datos bancarios de clientes a terceros, también llamados TPPs (Third Party Providers), favorecido por la entrada en vigor de la Segunda Directiva de Servicios de Pago, promoverá el crecimiento de los gigantes tecnológicos y las startups al permitirles el acceso a la información de los clientes bancarios y facilitando su intervención en el proceso de pagos, jugando de este modo un papel cada vez más importante en los servicios financieros.

- Se producirá un gran incremento en los niveles de inversión en compañías dedicadas a crear productos y soluciones específicas basadas en las tecnologías de registro distribuido, de las cuales el blockchain es un caso particular.

- La banca digital está en auge, el sector bancario tradicional está optando por adaptarse, hacer uso de estas innovaciones tecnológicas con el objetivo de desarrollar nuevos modelos de negocio con un mayor valor añadido, pues es la única forma de no quedar rezagado.

- El Internet de las cosas y los dispositivos inteligentes, basados en la inteligencia artificial, seguirán ampliando las posibilidades de realizar pagos y automatizar y simplificar las tareas cotidianas.

- Las técnicas biométricas y de tokenización contribuirán a aumentar la seguridad en los pagos, que es uno de los retos más importantes a los que se enfrenta el sector.

Estas transformaciones de los servicios financieros en general y de los pagos en particular basados en la digitalización y la tecnología traen cambios de orden económico, un aumento de la competencia en el sector, así como otros beneficios y transformaciones sociales, como por ejemplo servicios financieros más competitivos, más rápidos y personalizados y el acceso a los mismos de más segmentos de la población. Será necesario estar expectantes a cómo la tecnología, la ingeniería financiera y los nuevos hábitos de consumo configuran el sector en los próximos años. En este contexto, el papel de los gobiernos debe estar orientado a fomentar estos cambios garantizando un ecosistema seguro y un marco regulatorio capaz de optimizar los beneficios que supone el mundo fintech. Si bien es indiscutible que la revolución en el sector financiero no ha hecho más que empezar, provocará la aparición de nuevos operadores y productos y elevará los estándares de calidad de servicio de la banca en beneficio, sobre todo, de los usuarios.

\section{BIBLIOGRAFÍA}

AEFI (2017): Libro blanco de la regulación fintech en España, AEFI. Recuperado el 5 de abril de 2019 de https://www.asociacionfintech.es/recursos/libro-blanco-del-fintech/

Angelov A. (2019): El sistema financiero digital: los nuevos agentes, Documentos de trabajo 04/2019, Instituto Universitario de Análisis Económico y Social, Universidad de Alcalá de Henares, Alcalá de Henares.

Avendaño, E., Pérez, L. y Queizán, B. (2016): “Medios de pago, seguridad e identidad digital”, Papeles de Economía Española, n 149, pp. 127-143. 
Bank for International Settlements (BIS) (2018): Sound practices on the implications of fintech developments for banks and bank supervisors, Bank for International Settlements, Basilea.

BCG (2018): Global payments 2018: Reimagining the customer experience. Recuperado el 8 de abril de 2019 de https://www.bcg.com/publications/2018/global-paymentsreimagining-customer-experience.aspx

Bizum (2020): Entidades y Área de prensa. Recuperado el 19 de julio de 2020 de https://bizum.es/

Capgemini (2018): World Payments Report 2018. Recuperado el 4 de abril de 2019 de https://worldpaymentsreport.com/

Carbó, S. (2017): "Mitos y realidades de la digitalización financiera: los medios de pago como paradigma”, Mediterráneo Económico, n 29, pp. 139-149.

CNMC (13 de septiembre de 2018). Estudio sobre el impacto en la competencia de las nuevas tecnologías en el sector financiero (fintech), E/CNMC/001/18, Comisión Nacional de los Mercados y la Competencia.

Deloitte (2016): Disaggregating fintech: Brighter shades of disruption. Recuperado de https://www2.deloitte.com/content/dam/Deloitte/us/Documents/financial-services/usfsi-disaggregating-fintech-brighter-shades-of-disruption.pdf

Deloitte (2020a): Beyond COVID-19: New opportunities for fintech companies. Recuperado el 22 de julio de 2020 de https://www2.deloitte.com/content/dam/Deloitte/us/Documents/financial-services/usbeyond-covid-19-new-opportunities-for-fintech-companies.pdf

Deloitte (2020b): Fintech by the numbers. Recuperado el 30 de junio de 2020 de https://www2.deloitte.com/us/en/pages/financial-services/articles/the-next-phase-offintech-evolution.html

Facebook (2018): Acuerdo sobre transferencias interpersonales y cuenta de dinero electrónico de Facebook. Recuperado el 19 de julio de 2020 de https://esla.facebook.com/payments_terms/eu_regulated_payments

Fortumo (2019): Direct carrier billing in 2018: global market report by Fortumo, (12 de febrero de 2019). Recuperado el 10 de abril de 2019 de https://fortumo.com/blog/directcarrier-billing-in-2018-global-market-report-by-fortumo/

Funcas y KPMG (2018). Comparativa de la oferta de la banca vs. Fintech. Recuperado el 25 de marzo de 2019 de https://assets.kpmg/content/dam/kpmg/es/pdf/2018/06/comparativa-oferta-\%20bancafintech.pdf

Garijo, M. (2018): “Los bancos apuestan por el 'blockchain’ como solución para innovar y reducir costes”, Business Insider (28 de marzo de 2018). Recuperado el 15 de abril de 2019 de https://www.businessinsider.es/bancos-apuestan-blockchain-como-solucioninnovar-reducir-costes-193946

Google Pay (2019): Google Pay: Llega una mejor forma de pagar con Google. Recuperado el 22 de noviembre de 2019 de https://pay.google.com/intl/es_es/about/

Guersent, O. (2016): "La transformación digital de los instrumentos de pago", Papeles de Economía Española, n 149 , pp. 58-61.

Hill, J. (2018): Money: A medium of exchange, unit of account, and store of wealth. En J. Hill (Ed.): Fintech and the remaking of financial institutions (capítulo 3, pp. 41-68), Academic Press, Nueva York.

Huobi Research (2020): Global blockchain industry overview and prospects (2019-2020). Recuperado el 23 de junio de 2020 de https://medium.com/@huobiresearch/globalblockchain-industry-overview-and-prospects-2019-2020-ecb101e23970 
Kauffman, R. J., y Ma, D. (2015): “Contemporary research on payments and cards in the global fintech revolution”, Electronic Commerce Research and Applications, vol. 14, n 5, pp. 261-264.

KPMG (2019): The pulse of fintech 2018: biannual global analisis of investment in fintech. Recuperado el 22 de noviembre de 2019 de https://assets.kpmg/content/dam/kpmg/xx/pdf/2018/07/h1-2018-pulse-of-fintech.pdf

Observatorio de la Digitalización Financiera (2018): Comparativa de la oferta de la banca vs. fintech. Recuperado el 4 de abril de 2019 de https://www.funcas.es/_obsdigi_/

PayPal (2020): Venmo's total payment volume from 1st quarter 2017 to 1st quarter 2020 (in billion U.S. dollars). En Statista - El portal de las estadísticas. Recuperado el 23 de junio de 2020 de https://www.statista.com/statistics/763617/venmo-total-payment-volume/

Pérez, J. (2016): “La nueva era digital de los medios de pago”, Papeles de Economía Española, $\mathrm{n}^{\mathrm{o}} 149$, pp. 111-114.

PwC (2016): Financial services technology 2020 and beyond: Embracing disruption. Recuperado el 22 de noviembre de 2019 de https://www.pwc.com/gx/en/industries/financial-services/publications/financialservices-technology-2020-and-beyond-embracing-disruption.html

Richter, F. (2017). The Meteoric Rise of Venmo (6 de diciembre de 2017). Recuperado el 23 de junio de 2020 de https://www.statista.com/chart/12158/payment-volume-processedby-venmo/

Rjaibi, W. (2018): "Quantum computing and crypyography: What you need to know", IBM Think 2018 [online]. Recuperado el 10 de mayo de 2019 de: https://securityintelligence.com/quantum-computing-and-cryptography-what-youneed-to-know/

Romero, J.L. (2018): “Tecnología de registros distribuidos (DLT): una introducción”, Boletín económico, 4/2018, Banco de España, pp. 1-7.

Sánchez, J. (2019): WhatsApp se alía con la banca: la función de pagar desde su aplicación se empieza a extender, ABC (12 de abril de 2019). Recuperado de https://www.abc.es/tecnologia/moviles/aplicaciones/abci-whatsapp-alia-bancafuncion-pagar-desde-aplicacion-empieza-extender-201904101334_noticia.html

Schindler, J. (2017): Fintech and financial innovation: Drivers and depth, Finance and Economics Discussion Series, 2017-081, Board of Governors of the Federal Reserve System, Washington.

Schueffel, P. (2016): Taming the beast: A scientific definition of fintech, Institute of Finance, School of Management Fribourg, Switzerland.

Square (2020): Gross payment volume (GPV) of Square Inc. from 1st quarter 2015 to 1st quarter 2020 (in billion U.S. dollars). En Statista - El portal de las estadísticas. $\begin{array}{llllll}\text { Recuperado el } & 23 & \text { de junio de }\end{array}$ https://www.statista.com/statistics/575342/square-quarterly-payment-volume/

Statista (2019a): In-depth: FinTech 2019. Statista digital market outlook - Trend report. Recuperado el 23 de junio de 2020 de https:/www.statista.com/study/45600/statistareport-fintech/

Statista (2019b): Worldwide spending on blockchain solutions from 2016 to 2022, by region. $\begin{array}{llllll}\text { Recuperado el } 23 \text { de junio de } & 2020 \text { de }\end{array}$ https://www.statista.com/statistics/800561/worldwide-blockchain-solutions-spendingby-region/

Statista (2020a): Fintech report 2020. Statista digital market outlook - Market report. Recuperado el 22 de junio de 2020 de https:/www.statista.com/study/44525/fintechreport/ y https://www.statista.com/outlook/295/100/fintech/worldwide 
Statista (2020b): eCommerce report 2020. Statista digital market outlook - Market report. $\begin{array}{llllll}\text { Recuperado el } & 22 & \text { de } & \text { junio de }\end{array}$ https://www.statista.com/study/42335/ecommerce-report/ https://www.statista.com/outlook/243/100/ecommerce/worldwide

Statista (2020c): Global consumer survey 2020. Recuperado el 1 de julio de 2020 de https://www.statista.com/forecasts/1001382/mobile-payments-by-brand-in-spain $\quad \mathrm{y}$ https://www.statista.com/customercloud/global-consumer-survey

Tramazaygues, P. (2016): "Nuevo ecosistema digital en el sector de los pagos”, Papeles de Economía Española, no 149, pp. 78-92.

Twyp (2020): Usando Twyp y Mi tarjeta Twyp. Recuperado el 19 de julio de 2020 de https://www.twyp.com/

World Economic Forum (2016): The future of financial infrastructure. An ambitious look at how blockchain can reshape financial services. Recuperado el 14 de abril de 2019 de http://www3.weforum.org/docs/WEF_The_future_of_financial_infrastructure.pdf

Zelle (2020): Digital payment volume of Zelle from 3rd quarter 2017 to 4th quarter 2019 (in billion U.S. dollars). En Statista - El portal de las estadísticas. Recuperado el 23 de junio de 2020 de https://www.statista.com/statistics/871668/zelle-payment-volume-perquarter/ 